\title{
Scalability and Power Consumption of Static Optical Core Networks
}

\author{
Slavisa Aleksic ${ }^{(1)}$, Ward Van Heddeghem ${ }^{(2)}$, and Mario Pickavet ${ }^{(2)}$ \\ (1) Vienna University of Technology, Institute of Telecommunications, Favoritenstrasse 9-11/389, 1040 Vienna, Austria \\ (2) Department of Information Technology (INTEC) of Ghent University - IBBT, Gaston Crommenlaan 8, B-9050 Gent, Belgium \\ slavisa.aleksic@tuwien.ac.at,ward.vanheddeghem@intec.ugent.be,mario.pickavet@intec.ugent.be
}

\begin{abstract}
A large amount of traffic in core networks is highly aggregated and core nodes are interconnected by high-capacity links. Thus, most of the traffic demands in the core area can be accommodated by providing more or less static connections between ingress and egress nodes. In this paper, we describe and study three particular realizations of static optical core networks and compare them with the dynamic, packet switched architecture based on wavelength-division multiplexing (WDM) transmission and conventional electronic packet routers. We introduce an analytical model for estimating the average number of required switch ports for different network topologies in order to assess both scalability and power consumption of the considered network concepts. The results show that the concept of a static optically transparent core network promises high energy efficiency, and scalability to several tens of nodes.
\end{abstract}

\section{INTRODUCTION}

Core networks are on the top of the network hierarchy. They provide reliable and high-capacity connections between multiple regional network areas that aggregate and process traffic originating form a large number of users. Today, network elements within the core area provide transport, switching and traffic management functionality at several layers. In addition to highcapacity optical transmission links that are based on dense WDM technique, they usually comprise electronic equipment providing circuit switching (e.g., optical transport network - OTN and synchronous optical network - SDH/SONET), packet switching (e.g., Internet protocol (IP) switching) and a kind of label switching such as multiprotocol label switching (MPLS). In some cases, core nodes inspect and process each individual data packet, which can lead to huge processing overhead and non-deterministic delays. Such a complex and energyconsuming architecture is usually justified by the need for providing high flexibility, a high level of quality of service (QoS) and extended manageability. However, a static network providing reconfigurable paths has a simpler architecture and is able to guarantee low and deterministic end-to-end delays at the cost of a larger port count of switching fabrics and a slow reaction to changes in traffic demands. The term static is used here to contrast with the dynamic switched solutions outlined above.

The traffic in core networks is usually highly aggregated, so that traffic demands can be accommodated by providing more or less static connections between ingress and egress nodes. On the other hand, optical technologies are well suited for implementing highcapacity transmission and for providing semi-static connections with wavelength granularity. Optical switches capable of providing such reconfigurable lightpaths are already commercially available. Some examples of such switching devices are reconfigurable optical add-drop multiplexers (ROADMs) and optical cross connects (OXCs). A single optical fiber typically carries 40 or 80 wavelength channels at $10 \mathrm{Gbit} / \mathrm{s}$, $40 \mathrm{Gbit} / \mathrm{s}$ or even $100 \mathrm{Gbit} / \mathrm{s}$ each. Additionally, conventional outdoor optical cables usually comprise several tens or even more than hundred fibers (a standard number in metropolitan area is 144 fibers). All these facts suggest that sufficient capacity is either already available or can be made easily available except in long-haul transmission links, where lightening a new fiber is still difficult and expensive. However, utilization of the available lit capacity in long-haul intercontinental links is currently below 30\% [1] and about ten new submarine cables, i.e. hundreds of new fibers are installed every year, so both the number of fibers and the available bandwidth of long-haul transmission links are constantly increasing.

Technologies, architectures and requirements for reconfigurable slow optical switches such as ROADMs and OXCs have been extensively investigated in the past [2,3]. Optical technologies can be used to improve both performance and energy efficiency of core networks, for example by using energy-aware routing and wavelength assignment (RWA) algorithms [4] or by utilizing optical bypass together with efficient grooming strategies [5]. In general, applying a more static switching concept and utilizing slow optical switches promises a large reduction of the total network's power consumption [6].

The concept of using optical bypass to reduce power consumption has been studied before. The study by Shen [8] was one of the first to provide a detailed analysis on this subject. Vismara et al. compare in [9] the power consumption (and cost) of three architectures: a nonbypass, a bypass and a bypass-and-grooming architecture.

Our work, while also evaluating different variations on optical bypass to reduce power consumption, is different to these existing studies in a number of ways. First, we stretch the concept of optical bypass by suggesting an architecture that removes IP routers from the core altogether. Second, we propose and use a relatively simple analytical model that allows fast and easy estimation of the network's power consumption and port counts. This contrasts with the common use of ILPs and simulation techniques that are potentially more accurate but are also less transparent and more difficult to 
apply. Third, we not only perform case studies on reference networks (like NSFNET), but also consider minimum (ring) and maximum (mesh) physical connected networks to evaluate beyond case studyspecific results. Finally, we analyze the optical switching port count on its technical feasibility.

\section{CONSIDERED NODE AND NETWORK ARCHITECTURES}

This section describes the network concepts that we considered in this study. The four options include a fullydynamic approach, in which all core nodes are capable of paket switching in the electronic domain, and three realizations options with a more or less static circuit provisioning either in the electronic, optical or both domains: The corresponding node structures for each of the considered network concept are shown in Figure 1 and briefly described in the following.

- The node architecture of a fully dynamic core node providing packet switching in the electronic domain (DCN-WDM) is shown in Figure 1a. It comprises WDM transmission equipment and a large IP router capable of packet-by-packet processing and multiprotocol label switching (MPLS). The network based on such nodes is able to support low granularity of data and to provide a high link and network utilization by making use of the statistical multiplexing advantage.

- The second architecture, which is presented in Figure 1b, we refer to as the static core network node without optical bypass (SCN-WDM). Similar to DCN-WDM network nodes, all incoming optical signals are terminated at the node and processed in the electronic domain, but rather in a circuit-switched than in a packet-switched manner. Such a concept is known from pure electronic time-division multiplexed networks without packet switching capability (e.g. OTN and SONET/SDH).

- The static core network with optical bypass (SCNOB) architecture is the first of the two remaining node structures that comprise optical cross connects (OCXs) to forward optical signals directly in the optical domain, i.e., without optical/electrical/optical (OEO) conversion. Thus, the nodes are capable of performing the optical bypass. The SCN-OB node depicted in Figure 1c provides transparent optical connections through OXC for some selected wavelength channels, while the remaining channels are subject of $\mathrm{O} / \mathrm{E} / \mathrm{O}$ conversion. Note that grooming at the subwavelength level can be performed on the channels that are electronically processed.

- Finally, Figure 1d shows the static optically transparent core network node (SOTCN), in which the entire core (transit) traffic is transparently forwarded by the OXC, i.e., the transit traffic is $100 \%$ optically bypassed, while just the local (add/drop) traffic is $\mathrm{O} / \mathrm{E} / \mathrm{O}$ converted by means of $N_{n}-1$ transponders. Because we have done away with any electronic processing, no grooming can be performed, which can result in low link utilization.

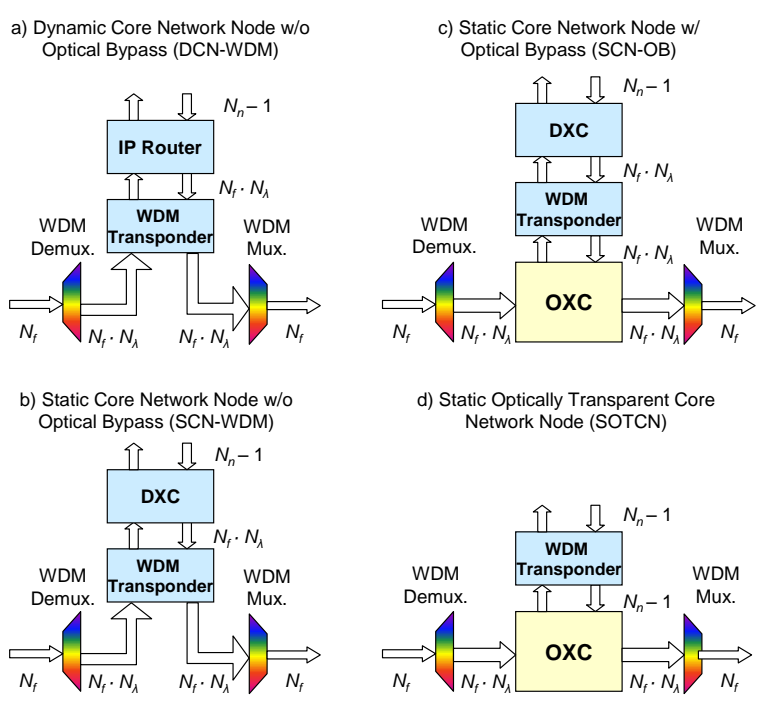

Figure 1. Architectures of four considered realizations of core network nodes: a) fully dynamic core node providing packet-by-packet processing in the electronic domain (DCN-WDM), b) static realization without optical bypass (SCN-WDM), c) static node providing optical bypass (SCN-OB), and d) static optically transparent node (SOTCN).
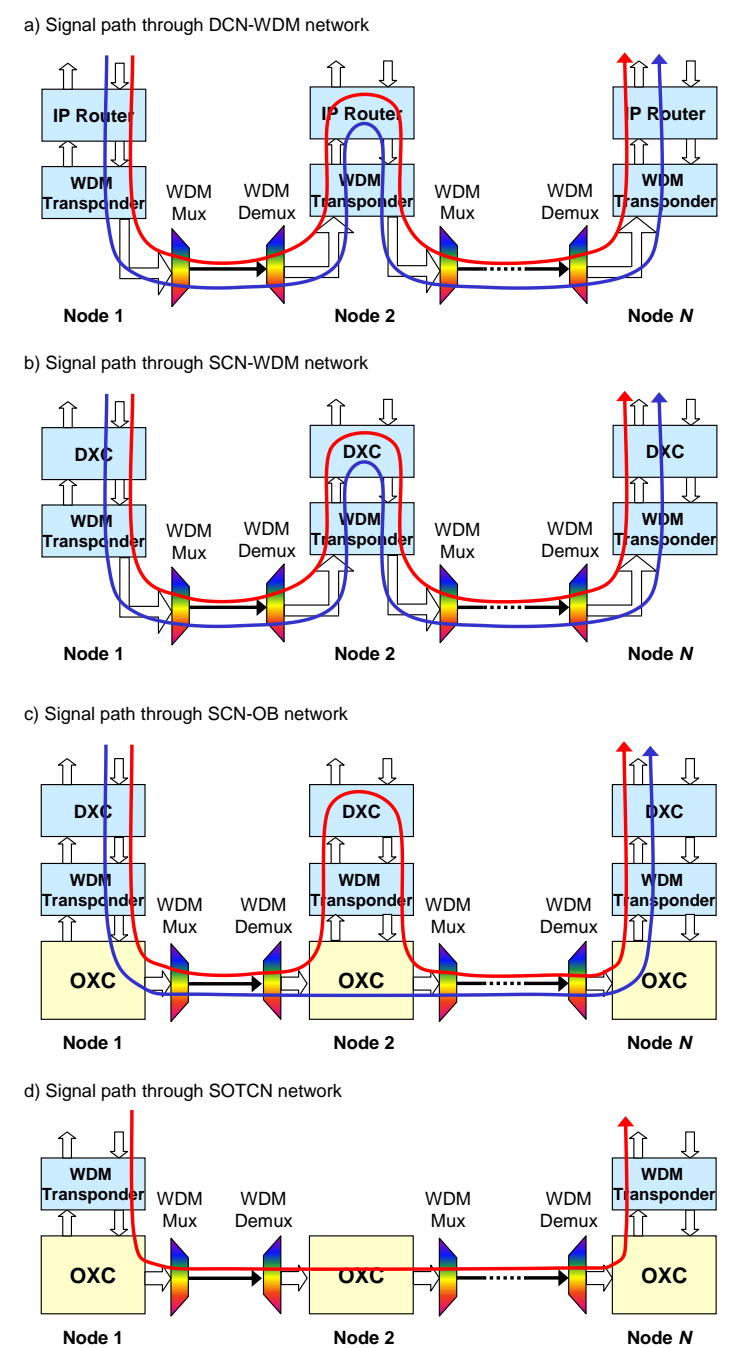

Figure 2. Typical signal paths through a) DCN-WDM, b) SCN-WDM, c) SCN-OB, and d) SOTCN core networks. 
To better clarify how the considered architectures are used in networks, we show typical signal paths in Figure 3. When observing this figure it becomes evident that in networks based on DCN-WDM and SCM-WDM, all channels are subject to $\mathrm{O} / \mathrm{E} / \mathrm{O}$ conversions at each traverse node, while networks using the SCN-OB architecture are able to provide optically transparent lightpaths on some channels. A SOTCN node never terminates transit channels. Thus the $\mathrm{O} / \mathrm{E} / \mathrm{O}$ conversion is only performed at network edges, while the core network is completely optically transparent.

The SOTCN architecture (Figure 1d) can be used to build static optically transparent core networks, which are based upon providing dedicated direct connections between each node pair in a static reconfigurable manner. The connections are established directly in the optical domain by setting bidirectional lightpaths between each ingress/egress node pair. The signal is transmitted between ingress and egress nodes in an optically transparent fashion without any switching or processing of data. At edge nodes, it is regenerated and monitored in the electronic domain. As indicated in Figure 3, a SOTCN utilizes reconfigurable optical nodes comprising OXCs, which provide dedicated connections between all core nodes with wavelength granularity. The OXCs can be realized using reconfigurable wavelength selective switches (WSSs). Electronic data processing and packet switching are completely removed from the core network. The transit traffic is neither terminated nor electronically processed by an intermediate node, unless there is a need for signal regeneration or monitoring. In a practical realization of SOTCN, it may be required to place SCNOB type nodes at some selected locations within the SOCTN network in order to provide an enhanced monitoring capability. Another solution could be to extend the SOTCN node architecture by adding simple signal monitoring elements to OXC ports. However, for the sake of simplicity and in order to see the effects of a pure SOCTN implementation, we do not consider regeneration and monitoring within the SOTCN architecture in this study.

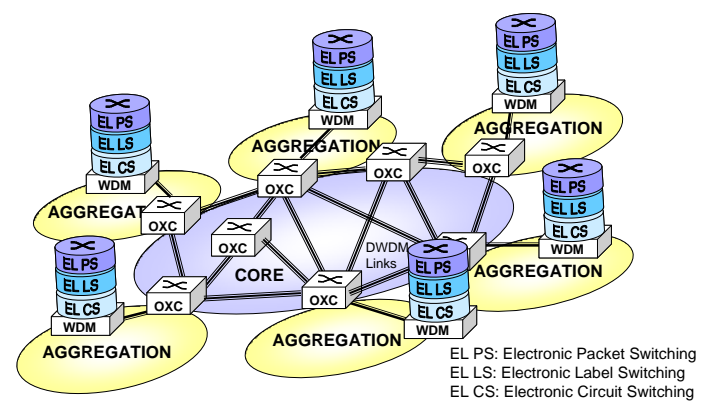

Figure 3. Static optically transparent core network (SOTCN).

Due to the fact that dedicated lightpaths are provided between all source-destination node pairs in a SOTCN, the topology at the wavelength layer is fully meshed as shown in Figure 4a, irrespective the physical (fiber) topology. In general, more than one direct connection between an ingress-egress node pair can be established to either provide a higher-capacity pipe or for protection, restoration and configuration purposes. However, we concentrate first on the basic concept by assuming only one dedicated lightpath between each node pair. The minimum and maximum physical connectivity that we consider in this study are double counter-rotating ring (Figure 4b) and full mesh topology (Figure 4c). In addition to the uniform minimum and maximum physical connected networks, we consider three irregular reference networks: NSFNet (i.e., NOBEL-US) with 14 nodes, PanEuropean 28 (i.e., NOBEL-EU) with 28 nodes and GERMANY50 with 50 nodes [10].
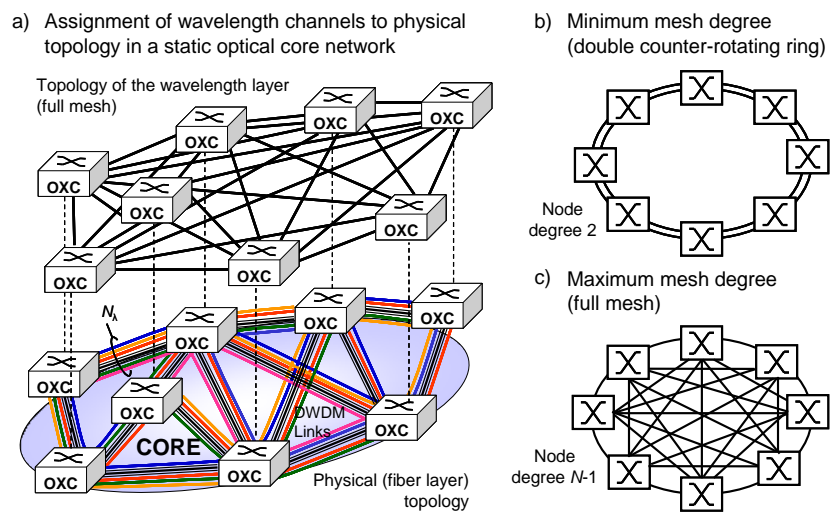

Figure 4. a) Fiber and wavelength layers of a static optically transparent core network; b) minimum and c) maximum physical connectivity considered in this study.

\section{SCALABILITY AND POWER CONSUMPTION ANALYSIS}

In the course of this study, we developed and aplied an analytical model for estimation of average (expectation) values of the main network design parameters for networks with regular (uniform) topology. On the base of these values and assuming uniform demands (i.e., the same demand from each node to each other node in the network) with a demand data rate $r_{d}$, we then calculated the average number of switch ports needed to accommodate the network total demand $D=N_{n}\langle d\rangle_{n}$, where $N_{n}$ is the number of nodes in the network and $\langle d\rangle_{n}$ denotes the average demands originating from one node. It can be easily shown that the number of links in a regular network composed of $N_{n}$ nodes with the average node degree, $\langle\delta\rangle_{n}$, is given by:

$L=\frac{N_{n}\langle\delta\rangle_{n}}{2}$

Note that the average node degree corresponds to the number of fiber ports $N_{f}$ as depicted in Figure 3. The maximum number of hops in the network can be calculated using the following semi-empirical formula:

$H_{\max }=\frac{N_{n}-1}{\langle\delta\rangle_{n}}$.

We obtain the average number of wavelength channels needed to accommodate the demands of a node without wavelength conversion capability using:

$\left\langle N_{\lambda, \text { node }}\right\rangle=\langle\delta\rangle_{n}\left(1+2+3+\cdots+H_{\max }\right)=\langle\delta\rangle_{n} \frac{\left(1+H_{\max }\right) H_{\max }}{2}$

Finally, the required average number of wavelength channels per link is: 


$$
\left\langle N_{\lambda, \text { link }}\right\rangle=\frac{N_{n}}{2 L}\left\langle N_{\lambda, \text { node }}\right\rangle=\frac{N_{n}\langle\delta\rangle_{n}\left(1+H_{\max }\right) H_{\max }}{4 L},
$$

and the average number of bidirectional OXC ports of a SOTCN network node can be calculated as:

$$
\left\langle N_{P, \text { OXC }}\right\rangle=\langle\delta\rangle_{n}\left\langle N_{\lambda, \text { link }}\right\rangle+N_{n}-1 \text {, }
$$

where $N_{n}-1$ represents the required number of bidirectional ports for accomodating local demands. Note that a bidirectional switch port is composed of two unidirectional ports.

In order to obtain the required number of OXC ports for a SCN-OB node, we need to introduce a new parameter defining the fraction of transit traffic being optically bypassed. Let $\beta$ be the portion of the total through traffic that is forwarded by the OXC, i.e., the traffic subject to optical bypass. Additionally, we define the grooming gain parameter, $g$, as the maximum number of demands that can be accomodated in a single wavelength channel, i.e., $g=r_{\lambda} / r_{d}$ where $r_{\lambda}$ is the channel data rate. When $\mathrm{O} / \mathrm{E} / \mathrm{O}$ conversion is provided and efficient traffic grooming is possible (i.e., $g$ is high), the capacity of wavelength channels can be fully utilized and the average number of required wavelengths per link is reduced by the factor $g$, i.e., $\left\langle N_{\lambda, \text { link,g }}\right\rangle=\left\langle N_{\lambda, \text { link }}\right\rangle / g$. For SCN-OB, we assume grooming capability and network nodes comprising $\langle\delta\rangle_{n}\left\langle N_{\lambda, l i n k, g}\right\rangle$ WDM transponders (see Figure 3b), of which $\beta \cdot\langle\delta\rangle_{n}\left\langle N_{\lambda, \text { link,g }}\right\rangle$ are inactive at each particular time and can be switched off. This is because the optically bypassed traffic does not need to be converted into the electronic domain. Thus, the number of required bidirectional OXC ports for the SCN-OB architecture is $2 \cdot\langle\delta\rangle_{n}\left\langle N_{\lambda, \text { link,g }}\right\rangle$ and the DXC used in the

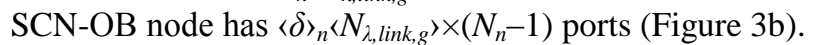

The architectures of SCN-WDM and DCN-WDM nodes are similar. They both require $\langle\delta\rangle_{n}\left\langle N_{\lambda, \text { link,g }}\right\rangle$ WDM transponders and do not comprise any OXC. An SCNWDM node makes use of a DXC with $\langle\delta\rangle_{n}\left\langle N_{\lambda, \text { link,g }}\right\rangle \times$ $\left(N_{n}-1\right)$ ports, while the DCN-WDM node employs an IP router of the same size.

For the irregular topologies of our three reference networks (see section II) the analytical model is not $100 \%$ accurate, so we applied a routing and wavelength assignment (RWA) algorithm to determine the required average number of wavelength channels per fiber. The RWA algorithm routes each of the uniform demands according to the shortest path algorithm through the network. The subsequent wavelength assignment is done using the First-Fit heuristic as described in [11]. Summarized, each wavelength on a fiber is numbered, and when searching for an available wavelength, the first available wavelength (from source to destination node) is selected. First-Fit is not an optimal heuristic, but is preferred in practice due to its low complexity and overhead. The power consumption analysis is carried out by calculating the numbers of active ports for different network realizations and topologies as described above and multiplying them by power consumption values per port for different equipment. For evaluation of power consumption we used the values specified in Table I, which are based on the values provided in [12].
TABLE I.

POWER CONSUMPTION VALUES

\begin{tabular}{|l|l|}
\hline Equipment & Power per port [W] \\
\hline \hline PLC-based WSS & 7 \\
\hline IP router (10G port, short range) & 100 \\
\hline WDM transponder 10G (short reach) & 40 \\
\hline WDM transponder 10G (long reach) & 50 \\
\hline DXC port 10G (short reach) & 40 \\
\hline
\end{tabular}

\section{RESUlTS AND DisCUSSIONS}

In this section, some selected results on scalability and power consumption of static optical networks are shown and discussed. Here, we assume the same channel and demand data rates for all networks, i.e., we set $r_{\lambda}=$ $10 \mathrm{Gbit} / \mathrm{s}$ and $r_{d}=2.5 \mathrm{Gbit} / \mathrm{s}$ that gives a grooming gain $g$ $=4$. The demands are uniform with a mean value per node of $N_{n}-1$. We assume an average portion of traffic being optically bypassed in SCN-OB nodes of $25 \%$ (i.e., $\beta=0.25$ ). Figure 5 shows the results on total network power consumption and switch port count for the four considered networks when varying the network size from $N_{n}=2$ to 100 . The curves are plotted for two regular topologies providing minimum and maximum connectivity (mesh) degree, i.e., for ring and full mesh topologies as shown in Figures $2 b$ and $2 c$, respectively. Note that the ring topology requires a larger port count than the full mesh topology because of the extra ports needed for the through traffic.

\section{A. Networks without restoration capability}

In general, the static optically transparent network (SOTCN) shows three to five times lower total power consumption than the full dynamic network for the same size and topology (see Figure 5a). For networks containing less than 55 nodes, SOTCN consumes less power than DCN-WDM irrespective of topology. For larger networks with more than 55 nodes, DCN-WDM in full mesh topology is less consuming than SOTCN arranged in a double counter-rotating ring structure. This is because SOTCN takes the advantage of low power consumption per port for smaller networks, while for very large networks in ring topology; SOTCN requires a very large number of switch ports as it is evident from Figure $5 \mathrm{~b}$, which leads to a high power consumption. For core links with higher utilization than assumed in our study, i.e., for a demand data rate higher than 1/4th of the channel data rate, SOTCN will be even more favorable over DCN-WDM, as higher utilization will result in increased power and port count for DCN-WDM but not for SOTCN. An interesting finding is that if $25 \%$ of the total incomming traffic in SCN-OB is bypassed by the OXC, SCN-OB is less energy efficient than SCN-WDM when assuming the full mesh topology and slightly more efficient in the ring topology. This is because the advantage of optical bypass is not utilized at all in a fully meshed network, while in the ring topology, the use of optical bypass results in a lower total power consumption because multi-hop lightpaths are possible. As one would expect, SOTCN provides the worst scalability among all 
considered networks, especially in the double counterrotating ring topology (see Figure 5b). With a switch port count of 100 , no more than 18 nodes can be connected in the ring topology. However, using the full mesh topology, static optically transparent networks with up to 50 nodes are realizable with 100 -port nodes. Since optical 3DMEMS switches with more than 1,000 ports are feasible [13], large static optical networks with up to 60 nodes in the ring and up to severeal hunderds of nodes in the full mesh topology could be possible.

In order to study the influence of the connectivity degree on both power consumption and port count, we define here a parameter MD (mesh degree) as the ratio of the average node degree of the network under consideration, $\langle\delta\rangle_{n}$, and the node degree of a full-meshed network having the same number of nodes as the considered network, $\langle\delta\rangle_{n \text {,mesh }}$, i.e.,

$$
M D=\left.\frac{\langle\delta\rangle_{n}}{\langle\delta\rangle_{n, \text { mesh }}}\right|_{N_{n}} \text {. }
$$

The calculated values of both total power consumption and required port count for various mesh degrees and two network sizes (30-nodes and 80-nodes networks) are shown in Figure 6. We can again observe that SOTCN is superior when considering power consumption. For mesh degrees below 0.2, SCN-WDM consumes slightly more power than SCN-OB. However, when the mesh degree increases above 0.2, SCN-WDM becomes less consuming and the difference in power consumption between SCN-OB and SCN-WDM increases. Note that for larger values of the parameter $\beta$ (i.e., for high percentages of traffic that is bypassed), SCN-OB would be more energy efficient than SCNWDM.

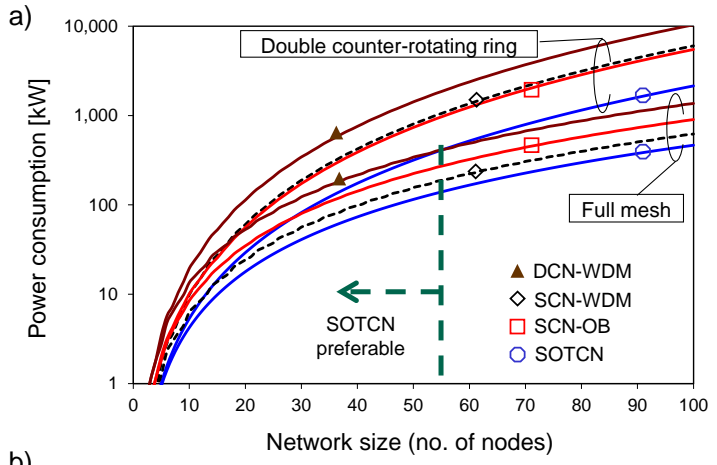

b)

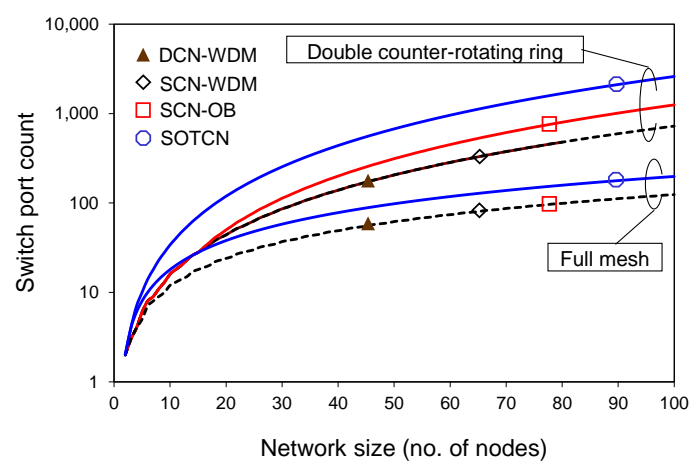

Figure 5. a) Total power consumption and b) required switch port count of the four considered networks versus network size.
When observing the required number of switch ports in Figure 6b, it becomes evident that if the mesh degree is decreased and the network size increased, the required switch port count for SOTCN increases rapidly. Especially for low values of the mesh degree below 0.2 and large networks with more than 50 nodes the required port count becomes very high. That implies a very high port count for large rings.
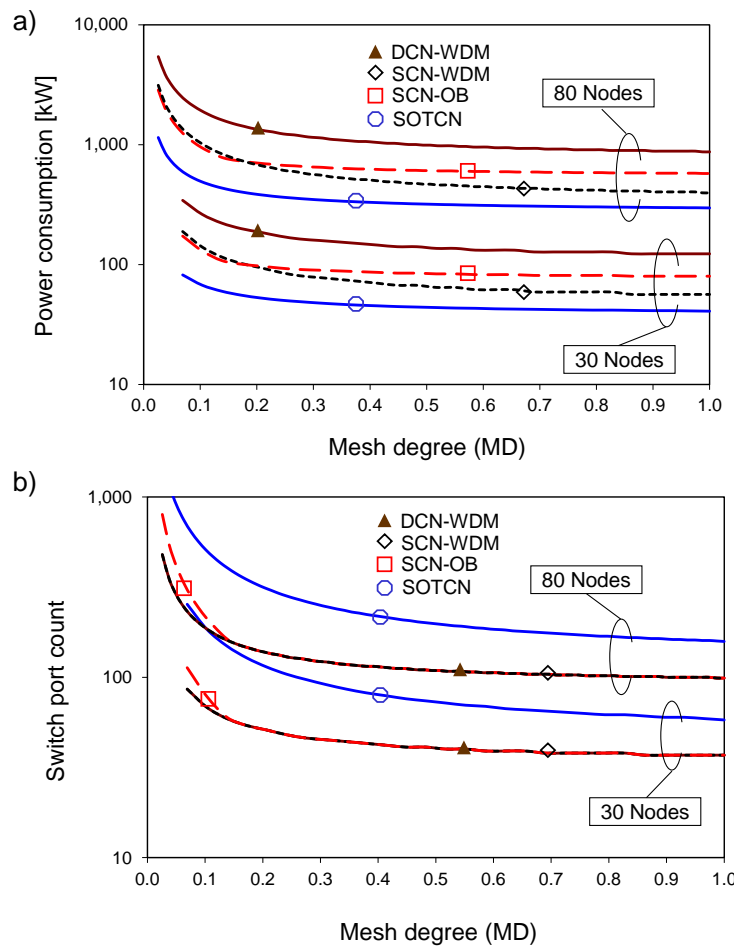

Figure 6. a) Total power consumption and b) required switch port count of the four considered networks versus mesh degree for two network sizes.

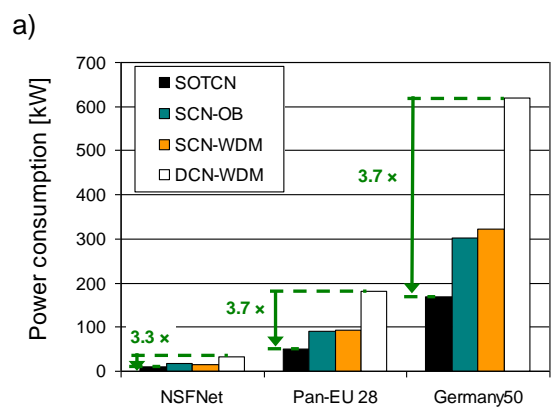

b)

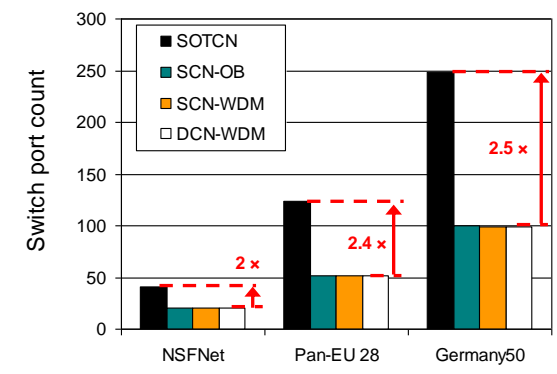

Figure 7. a) Total power consumption and b) required switch port count of the three considered examples of irregular reference networks. 
Finally, total power consumption and switch port count for the three considered examples of reference networks with irregular topologies are shown in Figure 7. The estimated achievable gain in power consumption for these networks when realized in a static optically transparent manner instead of using the DCN-WDM architecture is between 3.3 and 3.7. The increase in energy efficiency is paid by a larger switch port count, which has to be 2 to 2.5 times larger in SOTCN than in DCN-WDM. For mesh degrees below 0.1, 30-nodes SOTCN requires more switch ports than 80-nodes DCNWDM. However, for smaller networks with, for example, 30 nodes, the SOTCN port count remains below 250 irrespective of the mesh degree. For reference, the three reference networks have mesh degree 0.07 (GERMANY50), 0.11 (NOBEL-EU) and 0.23 (NOBELUS).

\section{B. Networks with restoration capability}

In order to ensure network survivability and to enable reconfiguration without any interruption of network operation, additional capacity has to be provided in the network. This additional capacity largely depends on what kinds of failures are considered, which restoration strategy is selected and what type of protection switch is used to redirect the affected traffic. We define here the network survivability in relation to single link failures. That means the network is designed robust enough to survive all single link failures. The additional capacity for restoration can be expressed as a fractional increase in the number of wavelength channels per link, i.e.,

$$
\left\langle N_{\lambda, \text { link }}^{R}\right\rangle=\left\langle N_{\lambda, \text { link }}\right\rangle(1+\langle R\rangle) \text {, }
$$

where $\langle R\rangle$ is the restoration factor determining the additional capacity needed for restoration and $\left\langle N_{\lambda, \text { link }}^{R}\right\rangle$ is the required average number of wavelength channels per link including the additional channels for restoration. The minimum required additional capacity for restoration can be obtained with an accuracy of about $17 \%$ when using the following semi-empirical formula [14]:

$$
\langle R\rangle \cong \frac{2}{\langle\delta\rangle_{n}} \text {. }
$$

For example, $\langle R\rangle$ becomes 1 for a ring network, as the node degree is 2 , and thus $100 \%$ extra link capacity is required. Note that this formula is a very good approximation for minimum required additional capacity in case of single link failures and strictly nonblocking cross connects.

The results for networks with additional capacity for restoration are shown in Figures 8, 9, and 10. In comparison to the results without restoration capability, the absolute values of both power consumption and switch port count are increased. Also the difference between the ring and the full mesh topology becomes larger. Here, SOTCN provides superior energy efficiency irrespective of network topology up to 30 nodes (see Figure 8a). However, it is still possible to realize a fully meshed SOTCN with approximately 50 nodes using OXCs with 100 ports (Figure 8b).

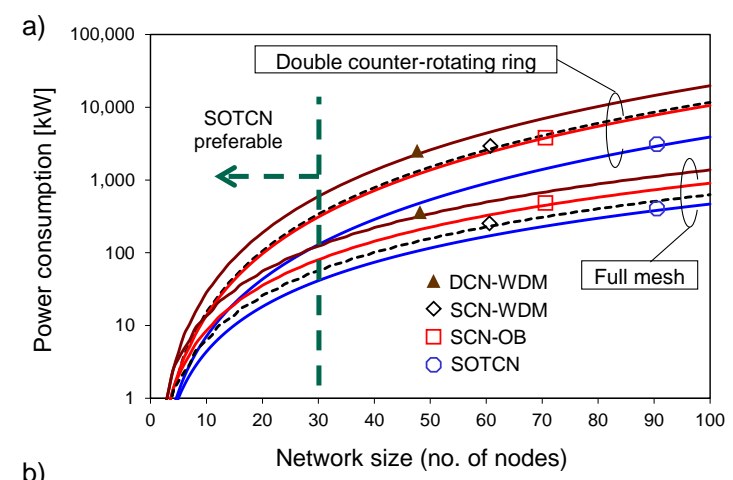

b)

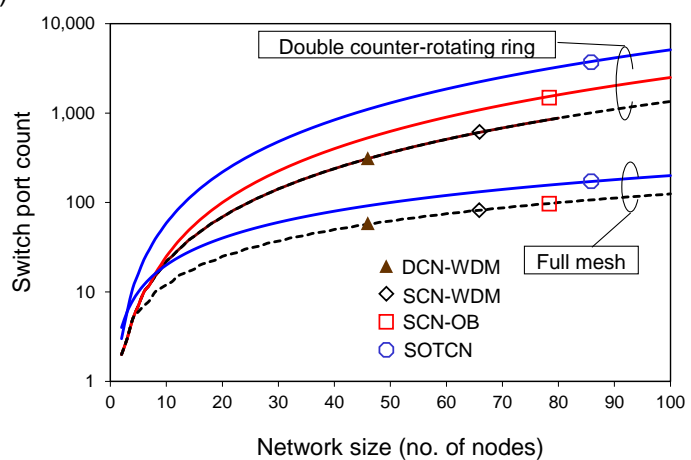

Figure 8. a) Total power consumption and b) required switch port count of the four considered networks versus network size when additional capacity is provided for restoration.
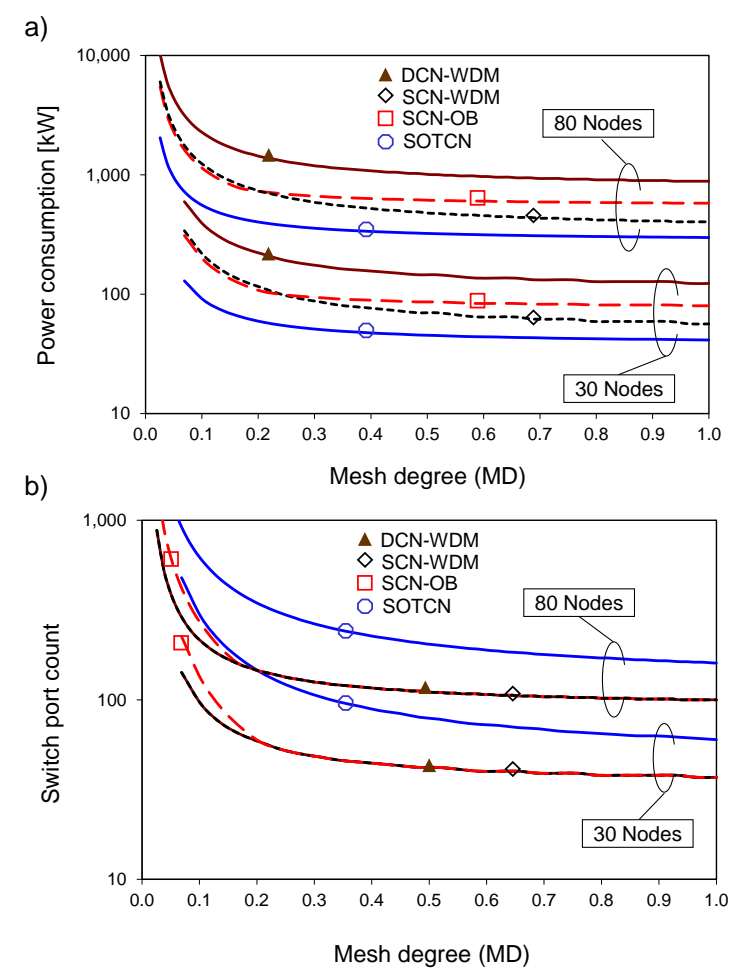

Figure 9. a) Total power consumption and b) required switch port count of the four considered networks versus mesh degree for two network sizes when additional capacity is provided for restoration.

Similar observations can be made when comparing the results presented in Figure 9 with those shown in Figure 6. The additional capacity for restoration has no influence on the intersection point between the curves representing the total power consumption of SCN-OB 
and SCN-WDM in Figure 9a. This is because the relative difference in power consumption between these two network realizations is primarily determined by the bypass factor $\beta$, which is assumed to be the same for both cases, namely $25 \%$.

However, if additional capacity for restoration is considered in the network design, the required number of ports for SOTCN increases faster than that for DCNWDM, so that a 30-nodes SOTCN requires a higher switch port count than a 80-nodes DCN-WDM already for a mesh degree below 0.2 (Figure 9b). Note that in the case without additional capacity for restoration, the intersection point is at 0.1 .

The total power consumption of the exemplary networks can be reduced by a factor between 3.7 and 4 when using the SOTCN architecture instead of DCNWDM. This is even a higher gain in power consumption than can be obtained for networks without restoration capability. However, the required number of switch ports is increased when comparing to the case without restoration capability (see Figure 10b).

a)

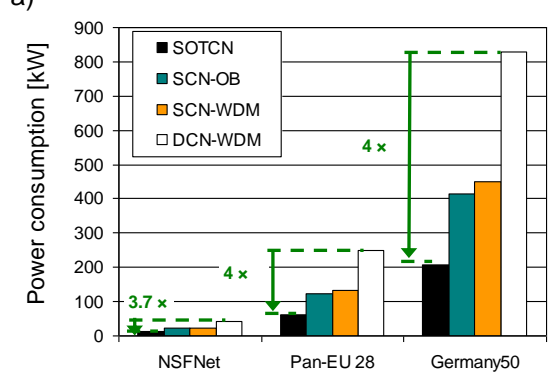

b)

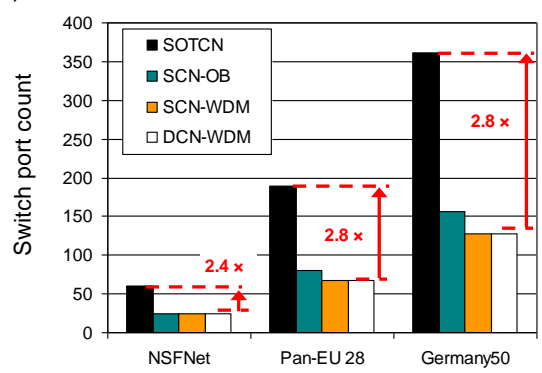

Figure 10. a) Total power consumption and b) required switch port count of the three considered examples of irregular reference networks with restoration capability.

\section{CONCLUSIONS AND FUTURE WORK}

In conclusion, we analysed three realization options for static optical core networks with regard to required number of switch ports and power consumption. The static networks are assessed by means of comparison with a dynamic network based on WDM transmission and conventional IP routers. An analytical model for a fast and easy determination of expectation values of the main network design parameters as well as for estimation of network's power consumption is described. Particular attention is paid to the concept of a static optically transparent core network where no digital cross connects are used. When applied to core networks with up to several tens of nodes and topologies with a sufficiently high connectivity degree, the proposed network concept promises significantly higher energy efficiency (in the order of $3 \mathrm{x}$ to $5 \mathrm{x}$ ) as well as lower and deterministic endto-end delays than the conventional, multi-layered, dynamic packet-switched network. These benefits come at the cost of higher - but still technically feasible switch port counts.

The further work will focus on a techno-economic study of the four considered network concepts, in order to assess viability of static optical core networks from the economic point of view. It would be also interesting to see what impact on energy consumption, technical feasibility and economic viability can be expected in case of unbalanced packet flows and increasing both channel and demand data rates. The latest is particularly important because $100 \mathrm{Gbit} / \mathrm{s}$ systems are starting to be introduced in core networks.

\section{ACKNOWLEDGMENT}

Part of this work has been supported by the STRONGEST project (grant agreement no. 247674) and the Network of Excellence TREND (grant agreement no. 257740) both funded by the European Community's Seventh Framework Programme FP7/2007-2013 under the mentioned grant agreements

\section{REFERENCES}

[1] TeleGeography, http://www.telegeography.com/

[2] S. Gringeri, et al., "Flexible architectures for optical transport nodes and networks", IEEE Communications Magazine, vol. 48, no. 7, pp. 40-50, Jul. 2010.

[3] J. Gripp, et al., "Architectures, Components, and Subsystems for Future Optical Packet Switches”, IEEE JSTQE, vol. 16, no. 5, pp. 1394-1404, Sept./Oct. 2010.

[4] P. Wiatr, et al., "Green lightpath provisioning in transparent WDM networks: Pros and cons”, IEEE ANTS, pp. 10-12, (2010).

[5] W. Van Heddeghem, et al., "Energy-Efficiency in Telecommunications Networks: Link-by-Link versus End-to-End Grooming”, ONDM 2010, Kyoto, Japan, pp. 1-6, Feb. 2010.

[6] S. Aleksic, "Analysis of Power Consumption in Future HighCapacity Network Nodes", OSA/IEEE JOCN, vol. 1, No. 3; pp. 245-258, August 2009.

[7] M. Fiorani, M. Casoni, S. Aleksic, "Performance and Power Consumption Analysis of a Hybrid Optical Core Node", OSA/IEEE JOCN, vol. 3 , Issue 6, pp. 502-513, June 2011.

[8] G. Shen and R. S. Tucker, "Energy-Minimized Design for IP Over WDM Networks", OSA/IEEE JOCN, vol. 1, no. 1, p. 176-186, (2009).

[9] F. Vismara, V. Grkovic, F. Musumeci, M. Tornatore, and S. Bregni, "On the energy efficiency of IP-over-WDM networks", IEEE Latin-American Conference on Communications, Sep. 2010, pp. 1-6.

[10] S. Orlowski, et al., "SNDlib 1.0-Survivable Network Design Library”, Networks, vol. 55, No. 3, pp. 276-286 (2010).

[11] H. U. I. Zang, "A Review of Routing and Wavelength Assignment Approaches for Wavelength- Routed Optical WDM Networks," Optical Networks Magazine, vol. 1 no.1, pp. 47-60, Jan. 2000.

[12] W. Van Heddeghem, F. Idzikowski, W. Vereecken, D. Colle, M. Pickavet and P. Demeester, "Power consumption modeling in optical multilayer networks", Photonic Network Communications (2012), DOI: 10.1007/s11107-011-0370-7

[13] R. Ryf et al., "1296-port MEMS transparent optical crossconnect with 2.07 petabit/s switch capacity", OFC 2001, Anaheim, CA, USA, paper PD28, (2001).

[14] S. K. Korotky, "Network Global Expectation Model: A Statistical Formalism for Quickly Quantifying Network Needs and Costs", IEEE JLT, Vol. 22, No. 3, pp. 703-722 (2004). 